\title{
The influence of fractal geometry on anomalous skin-effect in metal systems
}

\author{
Nikolay Torkhov ${ }^{1,2,3,}{ }^{*}$, Leonid Babak $^{2}$, Andrey Kokolov ${ }^{2}$, and Feodor Sheyerman ${ }^{2}$ \\ ${ }^{1}$ Research Institute of Semiconductor Devices, 634034, Tomsk, Russia \\ ${ }^{2}$ Tomsk State University of Control Systems and Radioelectronics, 634050, Tomsk, Russia \\ ${ }^{3}$ Tomsk State University, 634050, Tomsk, Russia
}

\begin{abstract}
The paper defines basic criteria of surface fractal geometry for $50 \Omega \mathrm{Au} / \mathrm{i}-\mathrm{GaAs}\{100\}$ coplanar microwave transmission lines, which influence on active resistance of their skin-layer and inductivity $L$. The local approximation limit $\mathrm{L}$ for active resistance $\mathrm{L}(R)$ is $\approx 800 \mathrm{um}$ and for inductivity $\mathrm{L}(L)$ is $\approx 400 \mathrm{um}$.
\end{abstract}

\section{Introduction}

At anomalous skin-effect, in a skin-layer with the thickness $\delta$, when $\delta<l_{\text {ball }}$ and $\delta<<v_{F} / \varpi$, there are spatial and frequency dispersions of microwave electromagnetic waves (microwave signal) which result in violation of the Ohm's law $(\mathbf{j} \neq \sigma \mathbf{E})$. Here $l_{\text {ball }}=v_{F} / \tau_{\text {ball }}$ is an average value of low-energy electron free (ballistic) path length (at normal conditions $l_{\text {ball }}$ is $\sim 600 \mathrm{~nm}$ [1]) defined by the time of electron ballistic transfer in homogeneous metal sample (ballistic time) $\tau_{\text {ball }} ; \varpi=2 \pi \mathrm{f}$ is a cyclic frequency; $v_{F}$ is a Fermi velocity. The increase of microwave signal frequency $f$ at normal and subnormal temperatures leads to decrease of the depth of microwave field penetration into conductor surface $\delta \sim \varpi^{-1 / 3}$ (lateral size of skin-layer), increase of its active resistance $R$, and decrease of transmission line inductivity $L$ [2]. In this case, at anomalous skin-effect the functional dependences $R=R(\mathrm{f})$ and $L=L(\mathrm{f})$ are largely defined by the state of the metal surface, while surface impedance $\zeta$ $(\operatorname{Re} \zeta=R)$ of its skin-layer is virtually independent of electrons' lattice scattering pattern [3].

The investigations of size (percolating) effects at direct and alternating electric currents in metal thin-film systems come mainly from the study of the influence of their lateral (perpendicular to $(\mathrm{x}, \mathrm{y})$ plane) sizes on the current flow peculiarities [4].

Meanwhile, the real metal films used in electronic device manufacturing have significant lateral (in $(\mathrm{x}, \mathrm{y})$ plane) irregularities as they consist of individual nanograins of monocrystals (crystallites) with the sizes of $20-500 \mathrm{~nm}$, separated from each other by resistive gaps - Mayadas-Shatzkes model [5]. In case when metal films are relatively thick (3-10 um), the size of their lateral irregularities can achieve hundreds micrometers [6], and the size of the normal ones (defined by the height of flatwise relief roughnesses) can achieve hundreds nanometers, which is comparable with skin-layer thickness at frequencies above $10 \mathrm{GHz}$ (e. g. for $\mathrm{Au} \delta(\mathrm{f}=20 \mathrm{GHz}) \approx 550 \mathrm{~nm}[7])$.

* Corresponding author: trkf@mail.ru 
Physical considerations reveal that such lateral irregularities can significantly influence on the motion of electrons in metal film skin-layer due to their scattering on grain boundaries as well as on surface roughnesses that for sure has an impact on the quality of microwave signal passage.

The spatial distribution of such irregularities in real metal systems is well described by the mathematical tool of fractal geometry. It is known [8] that the local approximation limits L, or linear sizes within which thin film metal systems exert properties of statistically fractal objects, can achieve values of hundreds micrometers that is comparable with the length of transmission microstrip lines in integral circuits.

Based on the above stated, and also taking into account the lack of contemporary science works devoted to the influence of lateral irregularities of surface and subsurface on propagation of electromagnetic microwaves inside them, it is possible to say that investigations in this sphere are of great research and practice interest. More specifically, it is important to study an impact of thin film fractal geometry on skin-effect peculiarities.

\section{Study subjects and experimental technique}

The investigation of the influence of Au surface geometry of microwave transmission coplanar lines (CPL) on their device characteristics in frequency range of $0.1-50 \mathrm{GHz}$ was carried out with the use of the notions of measure $M$, metric spaces $\mathfrak{R}(X, \rho)$ (consisting of some set $\mathrm{X}=\left\{x_{i}\right\}$ where the distance $\rho$ between any pair of elements with triangle, symmetry and zero distance axioms was defined), and normalized functional spaces $\mathbf{S}(\mathfrak{R}$, M) (hereinafter referred to as functional spaces) which made it possible to consider scalar and vector values, continuous functions and number sequences from the same point of view.

The subjects under investigation were the $50 \Omega$ coplanar lines with the same width $\mathrm{W}=50 \mathrm{um}$ and various length $l_{W}-100,200,400,800,1600$, and $3200 \mathrm{um}$. The lines were formed on $350 \mathrm{um} \operatorname{GaAs}\{100\}$ semi-insulating wafer by $3 \mathrm{um}$ Au plating with thin adhesive underlayer of vanadium (Fig. 1,a). The distance between signal and ground coplanar lines $S_{W}=36 \mathrm{um}$. The CML topological dimension was equal to one, $D_{T}=1$.
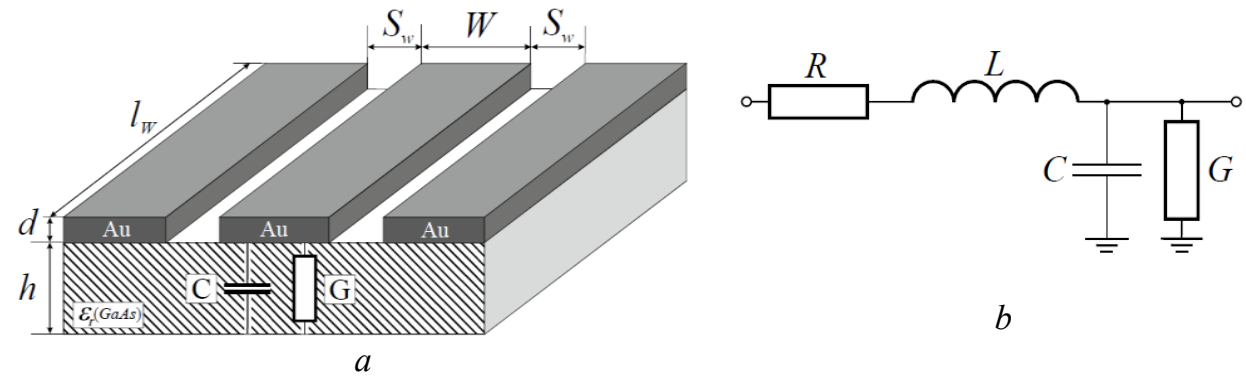

$b$

Figure 1. CML structure $-a$ ) and its small-signal equivalent circuit $-b$ ).

As far as the CML is formed by the assembly of various structural elements (ground and signal metal lines, semiconductor wafer), it is convenient to study its microwave parameters with the use of compact model in the form of equivalent circuit (EC) consisting of several equivalent elements and electric connections between them (Fig. 1,b). The passive components $R$ and $L$ describe directly serial resistance and inductivity of the CML, the components $C$ and $G$ correspond to capacity and conductivity of the wafer.

The measurement of metal film surface relief geometry $h(x, y)$ and phase contrast $\theta(x, y)$ was performed by means of the Solver-HV high-vacuum atomic force microscope (AFM) made by NT-MDT (Moscow, Zelenograd) with the use of the NSG10 cantilever. The 
rounding radius $r$ of the needle point plated with tungsten carbide $\left(\mathrm{W}_{2} \mathrm{C}\right)$ was $\approx 30 \mathrm{~nm}$. The AFM measurement was carried out in semi-contact scan mode using double-pass technique at resolution of 256 pixels both in vertical and horizontal sweeps.

The measurement of CML small-signal s-parametes in frequency range of $0.1-50 \mathrm{GHz}$ was performed with the use of the PNA-X N5245A vector network analyzer made by Keysight Technologies at the Summit 12000 semi-automatic probe station made by Cascade Microtech. Calibration of the measuring paths of the ports was carried out through s-parameters by means of pass-through (Thru), $50 \Omega$ load (Load), no load (Open), and shortcircuit (Short) matching. After that, the procedure of the measured s-parameter deembedding was conducted.

The Hausdorff dimension $D_{H}$ of the CML surface relief metric space $\mathfrak{R}$ was defined using a cube counting method. For self-similar objects, in local approximation $\left(l_{W}<\mathrm{L}\right)$ Hausdorff dimension is equal to fractal dimension $D_{f}(1)$, and in global approximation $\left(l_{W} \geq \mathrm{L}\right)$ it is equal to the object topological dimension $D_{T}$.

$$
D_{f}=D_{T}+D_{S}=1+\ln (\eta) / \ln (\zeta)
$$

where $D_{S}$ is a similarity dimension which is defined by estimation of relative increase of islets number $M$ (measures) by a factor of $\eta=M_{0} / M_{k}$ at relative decrease of measurement scale linear size (length $l_{W}$ ) by a factor of $\zeta=l_{W, 0} / l_{W, k}[9]$.

According to [10], the dimensions $D_{f}$ of functional spaces of active resistances $\mathbf{S}(\mathrm{M} \equiv R)$ and inductivities $\mathbf{S}(\mathrm{M} \equiv L)$ determined at $\mathfrak{R}$ are estimated by the same technique (1), but the role of the measures $\mathrm{M}$ is played by the functionals of CPL active resistances $M \equiv R$ and inductivities $M \equiv L$.
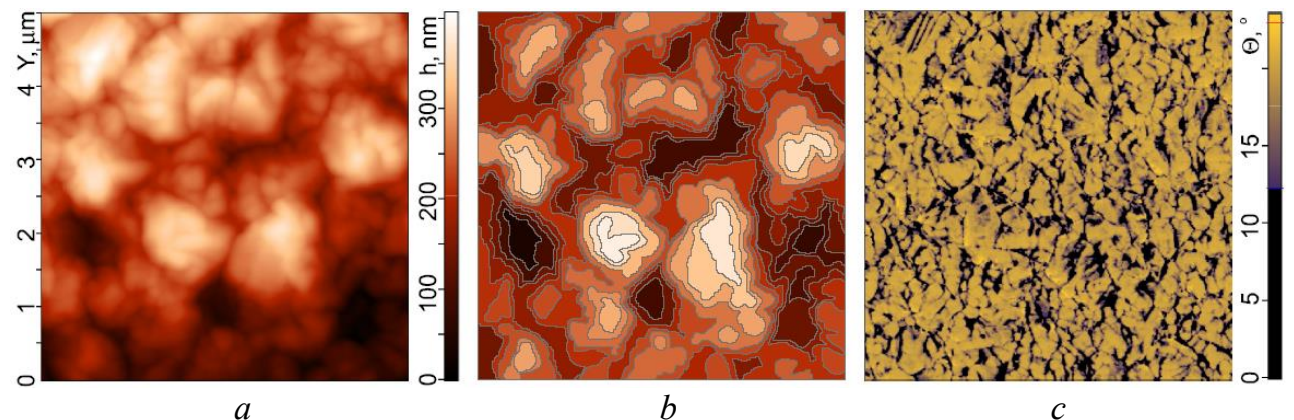

Fig. 2. The AFM images of surface relief $-a$ ), its contour image $-b$ ) and phase contrast $-c$ ).

\section{Experimental results}

As it is shown in Fig. 2, $a$, the size of relief irregularities of the CML $5 \times 5 \mathrm{um}^{2}$ surface area at the average value of roughnesses $\Delta \mathrm{h}>100 \mathrm{~nm}$ can distinctly be above, $\mathrm{h}>400 \mathrm{~nm}$, that is comparable with skin-layer thickness $(\delta(\mathrm{f}=20 \mathrm{GHz}) \approx 550 \mathrm{~nm})$. From general physical considerations, it is clear that electron scattering on such surface irregularities has significant influence on microwave signal propagation.

The pattern of relief irregularities distribution is revealed by the contour image of the surface area under investigation, obtained by tracing the irregularities (Fig. 2,b). The analysis of contour images shows that they are formed by smaller statistically self-similar elements of preceding similarity level put one into another.

The AFM images of phase contrasts $\theta(x, y)$ reveal the pattern of lateral positions of the grains (the geometry of granular substructure). According to statistical analysis, the CML 
gold film is formed by four sets of grains, which have the average sizes $d_{x}$ of something like $53,72,108$, and $133 \mathrm{~nm}$, that is significantly less than both skin-layer thickness

$$
d_{x}<<\delta(\mathrm{f}=20 \mathrm{GHz})
$$

and the average value of electron ballistic path length in homogeneous sample:

$$
d_{x}<<l_{\text {ball }} \text {. }
$$

The inequity (3) means that electron free path length $l_{\text {ball }}$ in the samples under investigation is limited by size of crystallites (grains). So, it is possible to say that for these samples $l_{\text {ball }} \approx d_{x}$. Therefore, in our frequency range $l_{\text {ball }}<<\delta$ and anomalous skin-effect of the studied CMLs becomes a normal one. It is obvious that the grain position geometry in CML gold film also has an influence on the electron scattering pattern and, subsequently, microwave signal propagation quality.

Fractal dimensions of metric spaces $\mathfrak{R}$ of surface relief $D_{f s}$ and granular substructure $D_{f g}$, defined by means of the cube counting method, are $2.11 \pm 0.08$ and $2.46 \pm 0.07$, correspondingly.

Therefore, the distributions of scattering centers in the form of relief irregularities and grain boundaries are described by different laws of fractal geometry which are expected to define integral characteristics of electron current in skin-layer and finally the quality of microwave signal propagation in the CML.
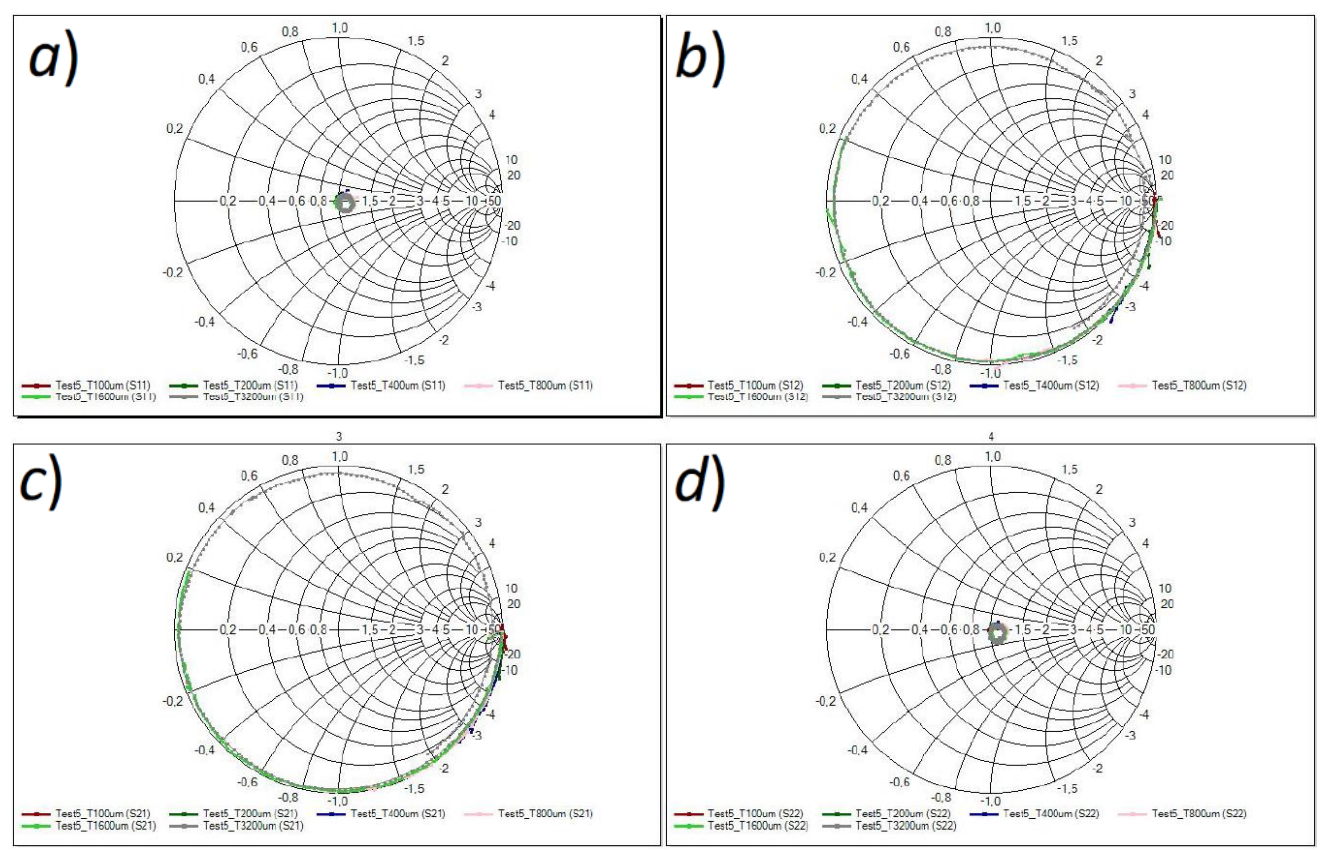

Fig. 3. Small-signal scattering parameters of CMLs with the length $l_{W}$ of 100, 200, 400, 800, 1600, and $3200 \mathrm{um}$, in the frequency range of $\left.\left.\left.0.1-50 \mathrm{GHz}: \mathrm{S}_{11}-a\right), \mathrm{S}_{12}-b\right), \mathrm{S}_{21}-c\right), \mathrm{S}_{22}-d$ ).

Let's see how the microwave signal passage relates to the CML fractal substructure. In Figure 3, there are Smith diagrams representing frequency dependences of small-signal sparameters of the CMLs under investigation having lengths $l_{W}$ of 100, 200, 400, 800, 1600, and $3200 \mathrm{um}$, in the range of $0.1-50 \mathrm{GHz}$. The central positions of $\mathrm{S}_{11}(\mathrm{f})$ and $\mathrm{S}_{22}(\mathrm{f})$ prove $50 \Omega$ surge impedance of the developed CMLs.

For the purpose of interpretation of the results obtained, let's consider rebuilt (extracted) parameters of the CML small-signal EC (Fig. 1,b) components (table 1). 
Table 1 shows clearly that up to some values $l_{W}<\mathrm{L} \approx 800 \mathrm{um}$ (in so-called local approximation) skin-layer serial resistance $R$ is disproportional to CML length $l_{W}$. In terms of serial resistances $R$, this reveals violation of the Ohm's law for the circuit section that could point out the existence of anomalous skin-effect. But the results of the AFM measurements prove the presence of the conditions for normal skin-effect realization in the studied CMLs in the frequency range used. At $l_{W} \geq \mathrm{L}$ (in global approximation) the values of serial resistance $R$ are virtually proportional to the length $l_{W}$.

In local approximation, at $l_{W}<\mathrm{L} \approx 400 \mathrm{um}$, size effects manifest themselves particularly clearly for the parameter $L$. In global approximation $\left(l_{W} \geq \mathrm{L}\right)$, inductivity $L$ grows virtually proportionally to the growth of the CML length $l_{W}$.

Therefore, it is possible to assume that size effects observed can be associated with fractal geometry of the CML metallization substructure.

Table 1. At frequency of $20 \mathrm{GHz}$.

\begin{tabular}{|c|c|c|c|c|c|c|}
\hline $\begin{array}{c}\text { Coplanar line } \\
\text { length } \\
l_{W}, \mu m\end{array}$ & \multicolumn{2}{|c|}{ Active resistance } & \multicolumn{2}{|c|}{ Inductivity } & Capacity & Conductivity \\
\cline { 2 - 7 } & $R, \Omega$ & $D_{S}(R)$ & $L, \mathrm{H}$ & $D_{S}(L)$ & $\mathrm{C}, \mathrm{fF}$ & $\mathrm{G}, 1 / \Omega$ \\
\hline 100 & 0.12 & 1.28 & $1.24 \times 10^{-10}$ & 1.42 & 1.41 & $1.44 \mathrm{E}-6$ \\
\hline 200 & 0.18 & 1.24 & $7.19 \times 10^{-10}$ & 1.14 & 2.90 & $3.00 \mathrm{E}-6$ \\
\hline 400 & 0.28 & 1.20 & $1.96 \times 10^{-9}$ & 1.03 & 5.76 & $5.81 \mathrm{E}-6$ \\
\hline 800 & 0.69 & 1.16 & $4.23 \times 10^{-9}$ & 0.99 & 12.03 & $1.11 \mathrm{E}-5$ \\
\hline 1600 & 1.65 & 1.06 & $8.12 \times 10^{-9}$ & 1.05 & 22.54 & $2.87 \mathrm{E}-5$ \\
\hline 3200 & 3.43 & 1.04 & $1.68 \times 10^{-8}$ & 1.03 & 44.72 & $5.01 \mathrm{E}-5$ \\
\hline
\end{tabular}

The determination of similarity dimensions $D_{S}$ of functional spaces of serial resistances $\mathbf{S}(R)$ and inductivities $\mathbf{S}(L)$ shows that there are values $D_{f}=1+D_{S}$ that differ significantly from topological ones $D_{T}=1$. This, together with linear dependences of $\ln \left(R_{0} / R_{k}\right)$ and $\ln \left(L_{0} / L_{k}\right)$ on $\ln \left(l_{W, 0} / l_{W, k}\right)$, reveals their fractal properties.

According to the results obtained for functional space of resistances $\mathbf{S}(R)$, in global approximation, when $l_{W} \geq \mathrm{L}$, the change of $l_{W}$ doesn't lead to significant change of $D_{f}(R)$ which is $\approx 2 \pm 0.08$ (table 1 ). This points out that the CML active resistance at microwave signal $(\mathrm{f}=20 \mathrm{GHz})$ is largely defined by $2 \mathrm{D}$ geometry of skin-layer surface relief irregularities distribution, which influences on lateral processes of electron scattering. The proximity of the values $D_{f s}=2.11$ of the metric space $\mathfrak{R}$ and $D_{f}(R)$ of the functional space $\mathbf{S}(R)$ proves that.

In local approximation, when $l_{W}<\mathrm{L}$, the decrease of $l_{W}$ leads to significant increase of $D_{f}(R)>2$ that points out a growing role of granular substructure in the process of electron scattering. The growing $D_{f}(R)$ values prove this.

For the functional space of inductivities $\mathbf{S}(L)$, the decrease of CML length $l_{W}$ down to the values of less than 400 um leads to significant increase of $D_{f}(L)$ values (from $D_{f}(L) \approx 2$ at $l_{W} \geq 400$ um to $D_{f}(L)=2.42$ at $l_{W}=100 \mathrm{um}$ ). This points out a significant role of $2 \mathrm{D}$ effects at $l_{W} \geq \mathrm{L}$ (global approximation) and $3 \mathrm{D}$ effects at $l_{W}<\mathrm{L}$ (local approximation) in CML inductivity building-up, that is not obvious. The proximity of the values $D_{f}(L)=2.42$ of the functional space $\mathbf{S}(L)$ and $D_{f g}=2.46$ of the grain metric space $\mathfrak{R}$ proves the fact that scales less than 400 um the CML inductivity is also affected by $3 \mathrm{D}$ peculiarities of granular substructure of the CML subsurface area.

\section{Conclusion}

The influence of gold plating surface fractal geometry on CML anomalous skin-effect, skin-layer active resistance $R$ and inductivity $L$ is evaluated. 
It is revealed that granular substructure of CML plating and irregularities of CML surface relief are the reasons for additional electron scattering on grain boundaries that at frequencies $\mathrm{f}>10 \mathrm{GHz}$ converts the anomalous skin-effect into the normal one.

Additionally, the lateral distribution of relief irregularities and granular substructure follows the fractal geometry laws that defines non-linear behavior of CML $R$ and $L$ parameters in local approximation at $l_{W}<\mathrm{L}$.

At this, in local approximation non-linear dependence of $R$ on $l_{W}$ is explained by the fractal geometry of surface relief and subsurface area of the CML. At the same time, in local approximation non-linear dependence of CML inductivity $L$ is provided by $2 \mathrm{D}$ and 3D geometry of the CML subsurface area.

The value of local approximation limits $L$ for the active resistance $L(R)$ is $\approx 800$ um and for the inductivity $L(L)$ is $\approx 400 \mathrm{um}$.

The study was conducted with support from the Ministry of Science and Higher Education of the Russian Federation. The unique ID of the work is 8.3423.2017/4.6.

\section{References}

1. V. O. Tin'kov, Length of free path of slow electrons in a solid depending on their Energy, Usp. Fiz. Met., 7, No. 2: 117-134 (2006)

2. A. V. Sokolov. Optical properties of metals. M.: Fizmatlit (1961)

3. M.I. Kaganov, P.Contreras. Theory of the anomalous skin effect in metals with complicated Fermi surfaces. Zh. Eksp. Teor. Fiz. 106, 1814-1829 (1994)

4. A. V. Latyshev, A. A. Yushkanov. Analytical description of the skin effect in metal with the use of a two parameter kinetic equation. Computational Mathematics and Mathematical Physics, 44:10, 1861-1872 (2004)

5. Lucas M.S.P. Electrical conductivity of thin metallic films with unlice surfaces. J. Appl. Phys., 36(5), 1632-1635 (1965)

6. Angadi M.A., Udachan L.A. Electrical properties of thin nickel films.- Thin Solid Films, v.79, N2, p.149-153 (1981)

7. D. K. Larson, in Physics of Thin Films, Ed. By M. H. Francombe and R. W. Hoffman Vol. 6 (Academic,New York 1971)

8. Antonets, I.V., Kotov, L.N., Nekipelov, S.V., Golubev E.A. et al. Nanostructure and Conductivity of Thin Metal Films. Tech. Phys. 49:306, 24-27 (2004)

9. A.F. Mayadas, M. Shatzkes, J.F. Janak. Electrical resistivity model for polycrystalline films: the case of specular reflection at external surfaces. Applied Physics Letters. V. 14, No. 11. P. 345-347 (1969)

10. N.A. Torkhov, L.I. Babak, A.A. Kokolov, A.S. Salnikov, I.M. Dobush, V.A. Novikov, I.V. Ivonin. Nature of size effects in compact models of field effect transistors. Journal of Applied Physics 119, 094505, doi: 10.1063/1.4942617 (2016)

11. N.A. Torkhov, L.I. Babak, A.A. Kokolov, F.I. Sheerman. Proceedings of the 29th International Conference «Microwave \& Telecommunication Technology» (CriMiCo'2019). Sevastopol, Russian Federation, September 8-14 (2019)

12. N. A. Torkhov, V. A. Novikov. Fractal geometry of the surface potential in electrochemically deposited platinum and palladium films. Semiconductors, 43(8), 1071-1077 (2009)

13. J. Feder. Fractals. M.: Mir (1991)

14. N.A. Torkhov. Sheet Resistance of TiAlNiAu thin film metallization of ohmic contacts to nitride semiconductor structures. Semiconductors, 53(1), 28-36 (2019) 